\title{
Salivary exRNA biomarkers to detect gingivitis and monitor disease regression
}

\author{
Karolina E. Kaczor-Urbanowicz ${ }^{1,2 *}$ (D) | Harsh M. Trivedi ${ }^{3 *}$ | Patricia O. Lima ${ }^{1,4}$ | \\ Paulo M. Camargo ${ }^{5}$ | William V. Giannobile ${ }^{6}$ (D) | Tristan R. Grogan ${ }^{7}$ | \\ Frederico O. Gleber-Netto ${ }^{8}$ | Yair Whiteman ${ }^{9}$ | Feng Li $^{1}$ | Hyo Jung Lee ${ }^{10}$ | \\ Karan Dharia ${ }^{11}$ | Katri Aro ${ }^{1}$ | Carmen Martin Carreras-Presas ${ }^{12}$ (D) | Saarah Amuthan ${ }^{11}$ | \\ Manjiri Vartak $^{11}$ | David Akin ${ }^{1}$ | Hiba Al-adbullah ${ }^{11}$ | Kanika Bembey ${ }^{11}$ | Perry R. Klokkevold ${ }^{5}$ | \\ David Elashoff $^{7}$ | Virginia M. Barnes ${ }^{13}$ | Rose Richter ${ }^{13}$ | William DeVizio ${ }^{13}$ | \\ James G. Masters ${ }^{3}$ | David T. W. Wong ${ }^{1}$
}

${ }^{1}$ UCLA School of Dentistry, Center for Oral/Head \& Neck Oncology Research, University of California at Los Angeles, Los Angeles, California

${ }^{2}$ Section of Orthodontics, UCLA School of Dentistry, University of California at Los Angeles, Los Angeles, California

${ }^{3}$ Early Research Oral Care, Colgate Palmolive Co., Piscataway, New Jersey

${ }^{4}$ Department of Physiological Sciences, Piracicaba Dental School, University of Campinas, Piracicaba, São Paulo, Brazil

${ }^{5}$ Section of Periodontics, UCLA School of Dentistry, University of California at Los Angeles, Los Angeles, California

${ }^{6}$ Department of Periodontics and Oral Medicine, School of Dentistry, University of Michigan, Ann Arbor, Michigan

${ }^{7}$ Department of Biostatistics, University of California at Los Angeles, Los Angeles, California

${ }^{8}$ Medical Genomics Laboratory, Centro Internacional de Pesquisa e Ensino (CIPE), AC Camargo Cancer Center, São Paulo, Brazil

${ }^{9}$ UCLA School of Dentistry, Center for Esthetic Dentistry, University of California at Los Angeles, Los Angeles, California

${ }^{10}$ Section of Dentistry, Department of Periodontology, Seoul National University Bundang Hospital, Seoul, Korea

${ }^{11}$ UCLA School of Dentistry, University of California at Los Angeles, Los Angeles, California

${ }^{12}$ Faculty of Biomedical Sciences, Adult's Dentistry Department, Universidad Europea de Madrid, Madrid, Spain

${ }^{13}$ Clinical Research Oral Care, Colgate Palmolive Co., Piscataway, New Jersey

\section{Correspondence}

David T. W. Wong, UCLA School of

Dentistry, Center for Oral/Head \& Neck

Oncology Research (COOR), 10833 Le Conte

Avenue, Los Angeles, CA 90095-1668.

Email: dtww@ucla.edu

and

William DeVizio and James G. Masters,

Advanced Technology Oral Care, Colgate

Palmolive Co., 909 River Road, Piscataway,

NJ 08855.

Emails: william_devizio@colpal.com;

jim_masters@colpal.com

Funding information

This work was supported by a research grant from the Colgate-Palmolive Company-

Protocol Number ERO-2015-SAL-03-HT and the Public Health Service (PHS) grants from the National Institutes of Health (NIH): UH3 TR000923 and R90 DE022734.

\begin{abstract}
Aim: This study tests the hypothesis that salivary extracellular RNA (exRNA) biomarkers can be developed for gingivitis detection and monitoring disease regression.

Materials and Methods: Salivary exRNA biomarker candidates were developed from a total of 100 gingivitis and non-gingivitis individuals using Affymetrix's expression microarrays. The top 10 differentially expressed exRNAs were tested in a clinical cohort to determine whether the discovered salivary exRNA markers for gingivitis were associated with clinical gingivitis and disease regression. For this purpose, unstimulated saliva was collected from 30 randomly selected gingivitis subjects, the gingival and plaque indexes scores were taken at baseline, 3 and 6 weeks and salivary exRNAs were assayed by means of reverse transcription quantitative polymerase chain reaction.
\end{abstract}


Results: Eight salivary exRNA biomarkers developed for gingivitis were statistically significantly changed over time, consistent with disease regression. A panel of four salivary exRNAs [SPRR1A, Inc-TET3-2:1, FAM25A, CRCT1] can detect gingivitis with a clinical performance of 0.91 area under the curve, with $71 \%$ sensitivity and $100 \%$ specificity.

Conclusions: The clinical values of the developed salivary exRNA biomarkers are associated with gingivitis regression. They offer strong potential to be advanced for definitive validation and clinical laboratory development test.

\section{KEYWORDS}

biomarkers, exRNA, gingivitis, monitoring, saliva

\section{1 | INTRODUCTION}

Periodontal diseases are the most common inflammatory diseases in humankind (Kassebaum et al., 2017). Gingivitis, the reversible form of the disease, is induced by the accumulation of bacterial biofilm that can be treated with professional biofilm removal and improvement in oral hygiene (Kim, Kim, \& Camargo, 2013). If left untreated, gingivitis may progress to an irreversible form-periodontitis that involves bone and attachment loss. Periodontitis can advance to the point that it induces tooth mobility and tooth loss (Kim et al., 2013). It may also adversely influence systemic health via cardiovascular diseases (Kim et al., 2013), ischemic stroke (Grau et al., 2004) or cancers (Binder Gallimidi et al., 2015; Mitsuhashi et al., 2015; Rajesh, Thomas, Hegde, \& Kumar, 2013). The common risk factors for periodontitis could be divided into inherited (e.g. genetic variants), and those that are acquired (e.g. socioeconomic factors, poor oral hygiene, cigarette smoking and diabetes) (Chapple et al., 2017).

The quest to develop salivary biomarkers for periodontal diseases has been elusive (Giannobile et al., 2009). Despite the scientific acceptance of salivary biomarkers for the detection of gingivitis (Henskens, Van Der Velden, Veerman, \& Nieuw Amerongen, 1993; Kinney et al., 2011; Lee et al., 2012; Morelli et al., 2014; Shaila, Pai, \& Shetty, 2013), the absence of definitively validated biomarkers did not allow for such technology to receive regulatory approval and translating this diagnostic test to move into clinical practice. Presently, the gold standard for early detection and diagnosis for gingivitis includes a comprehensive periodontal exam along with clinical examination (i.e. redness, swelling or gingival bleeding) performed during dental examinations. Unfortunately, the absence of pain in gingivitis often renders individuals unaware of their pathological gingival condition, particularly if they do not have to visit the dentist regularly (Kim et al., 2013). While very helpful, clinical parameters do not provide full information about the current status of the disease activity, patient-specific variations, do not predict non-responders for treatment therapy and those who are "at risk" for disease progression (Ebersole, Nagarajan, Akers, \& Miller, 2015). Thus, it is desirable to develop objective and scientifically credible biomarkers for early detection and monitoring of periodontal diseases, especially

\section{Clinical Relevance}

Scientific rationale for the study: Presently, the gold standard for diagnosis of gingivitis includes mainly clinical examination. As a companion diagnostics, scientifically based evaluation of gingivitis would be very beneficial as it is the most prevalent inflammatory disease in humans.

Principal findings: Principles of prospective study design (PRoBE) and rigorous clinical classification of gingivitis led to the development and validation of salivary exRNA biomarkers for gingivitis detection that are associated with disease regression. Discriminatory salivary exRNA biomarkers were developed for gingivitis detection. Four salivary exRNA panels can discriminate gingivitis from healthy periodontium with a performance of 0.91 AUC, with $71 \%$ sensitivity and $100 \%$ specificity.

Practical implications: Salivary exRNA markers can be objective molecular indicators of periodontal health for disease detection as well as gingivitis regression.

that many of the affected cases are left undiagnosed and untreated (Albandar \& Kingman, 1999; Tomar \& Asma, 2000).

The applications of such biomarkers could be multiple. Firstly, they might be used by community at large as self-administered point-of-care (POC) tests to screen for periodontal diseases, thus making people aware of the pathology in their oral cavities and to encourage them to search for a dental care. The chairside POC devices will require minimal clinical training and resources, lead to simpler, more cost-effective and less intensive treatment (Giannobile, 2012). Due to close proximity of saliva to periodontal tissues and its easy collection, saliva constitutes a valuable diagnostic source, specifically as its collection is easy, repeatable and can be performed by auxiliary personnel. In addition, the salivary biomarkers present an attractive tool for quantitative 
and qualitative objective evaluation and monitoring of gingivitis for both scientists and dentists. The use of salivary-based POCs would also enable the underserved communities an improved access to oral health programmes as well as offers the ability to identify and monitor the patients at risk (Giannobile, 2012). Lastly, the dental, pharmaceutical and oral health care companies could use them for optimizing the pharmaceutical efficiency such as adequate selection of target patients, optimal dose selection and to assess the performance of the current active compounds in the development of their new oral hygiene products such as toothpastes, mouthwashes, etc.

This study addresses the unmet clinical need of assessing host factors as a companion diagnostics to detect gingivitis and monitor its treatment response.

\section{2 | MATERIALS AND METHODS}

\section{1 | Ethics statement}

The sample collection was approved by the Institutional Review Board (IRB) at the University of California at Los Angeles (UCLA) for both the discovery phase (IRB\#10-000505) and the clinical stage (IRB\#15-001957) of the study. Each participant had to sign the informed consent.

\section{2 | Discovery phase}

Based on the prospective-specimen-collection and retrospectiveblinded-evaluation (PRoBE) study design (Pepe, Feng, Janes,
Bossuyt, \& Potter, 2008), saliva was collected prospectively from 750 human subjects at the UCLA School of Dentistry. The inclusion and exclusion criteria are presented in Figure 1. Subjects were classified as healthy or gingivitis according to research and clinical criteria (Figure 1).

Prior to a clinical evaluation, unstimulated whole saliva was collected for every subject. Subjects were asked to avoid oral hygiene measures, eating, drinking or gum chewing at least $1 \mathrm{hr}$ prior to saliva collection. All subjects rinsed with tap water (10 $\mathrm{ml})$ for $30 \mathrm{~s}$ about $10 \mathrm{~min}$ prior to saliva collection and expectorated. Clinical samples were collected into sterile tubes, lasting 5-10 min per collection (at least $5 \mathrm{ml}$ of saliva), and kept on ice through the entire process. All samples were processed, around $1 \mathrm{hr}$ after collection. Samples were firstly centrifuged in a refrigerated centrifuge at $2,400 \times \mathrm{g}$ for $15 \mathrm{~min}$ at $4^{\circ} \mathrm{C}$, and the supernatant was processed immediately for the concurrent stabilization of proteins and RNA by the inclusion of a protease inhibitor cocktail (aprotinin, 3-phenylmethylsulfonyl fluoride [3-PMSF] [Sigma-Aldrich, St. Louis, MO, USA], sodium orthovanadate [Na3VO4] [SigmaAldrich] RNase inhibitor (Invitrogen SUPERase.In RNase Inhibitor [Thermo Fisher Scientific, Austin, TX, USA]) based on our saliva standard operating procedure (Henson \& Wong, 2010). These samples were aliquoted into smaller cryo-vials, labelled and frozen at $-80^{\circ} \mathrm{C}$.

Afterwards, salivary exRNA was extracted using the RNeasy Micro Kit [Qiagen, Germantown, MD, USA] including DNase I digestion. Samples with good purity QC (OD 260/280 1.8) were profiled using the GeneChip Human Transcriptome Affymetrix HTA 2.0 expression arrays [Thermo Fisher Scientific].

\section{Study design}

\section{Enrollment \\ prospective collection of saliva from 750 subjects (PRoBE design) \\ Selection criteria}

Research criteria: Healthy (non-periodontal disease): $\mathrm{MBI}<5 \%$ $\&$ PPD $<4$ mm; Gingivitis: $\mathrm{MBI}>5 \%$; PPD $<4 \mathrm{~mm}$.

Clinical criteria: Examination of the entire dentition, for six sites per tooth (mesio-buccal, mid-buccal, disto-buccal, mesio-lingual, mid-lingual, and disto-lingual), PI, visual signs of gingival tissue inflammation and radiographic bone levels Inclusion criteria: $\geq 20$ teeth, no periodontal treatment or antibiotic therapy 3 months prior to the investigation, no long-term use of anti-inflammatory drugs

Exclusion criteria: Smoking, metabolic bone diseases, autoimmune diseases, unstable diabetes or post-menopausal osteoporosis, pregnancy

\section{Discovery phase}

50 Gingivitis and 50 periodontally healthy subjects (taken from the enrollment phase)

\section{Selection criteria}

Randomisation criteria: Periodontal status (gingivitis/healthy), age, gender

\section{Validation phase}

30 New gingivitis patients

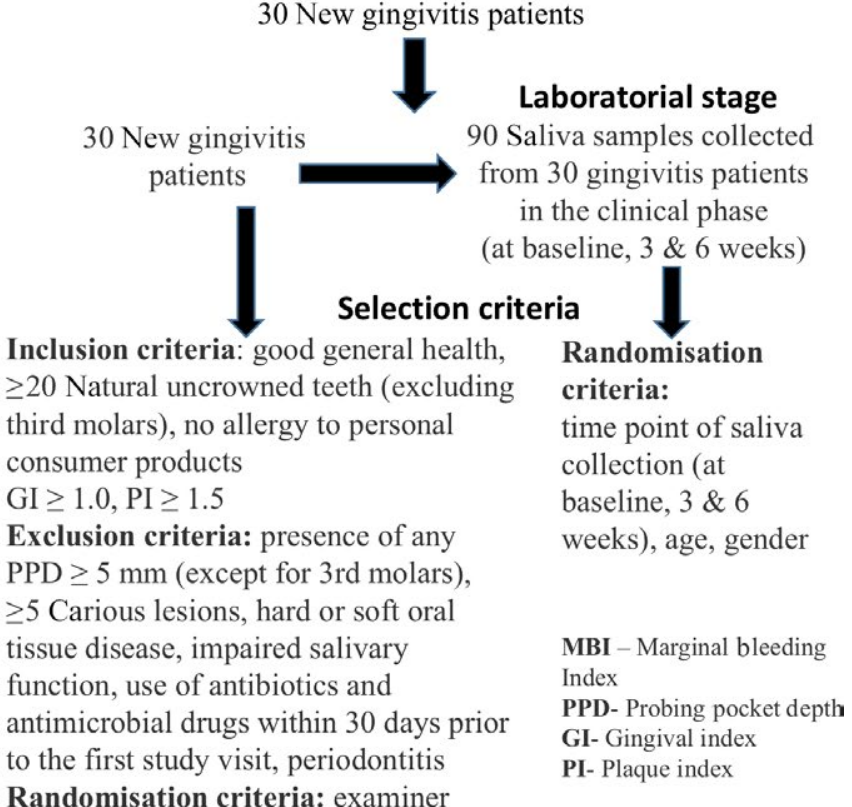

FIGURE 1 Study design: flow chart 


\section{3 | Pilot clinical research study}

The pilot study consisted of two stages: clinical and laboratory.

\subsection{1 | Clinical stage}

For analysis of saliva samples, 30 volunteers (aged 18-65 years), diagnosed with gingivitis, were included in this study. Figure 1 presents the inclusion and exclusion criteria. The duration of this project was 7 weeks (including 1 week pretrial training "washout" period to check if there are any allergic reactions to toothpaste's ingredients) and involved the same treatment protocol for gingivitis such as brushing with toothpastes containing 1,000 p.p.m. sodium monofluorophosphate (Colgate-Palmolive Company, New York, NY, USA). Each volunteer was instructed to brush the whole mouth with a full ribbon of the toothpaste for 1 min twice daily. The subjects expectorated and rinsed with water. Subjects returned for a clinic visit at baseline (B), 3 and 6 weeks to donate saliva samples prior to the clinical examination. The protocol for collection and processing of saliva was the same as described previously (discovery phase), but saliva was collected only in the mornings and immediately frozen at $-20^{\circ} \mathrm{C}$ for about $3-4 \mathrm{hr}$ until further analysis.

\subsection{2 | Laboratory stage}

\subsubsection{1 | Validation phase}

The direct saliva transcriptome analysis For the validation phase, the direct saliva transcriptome analysis (DSTA) was performed that uses cell-free saliva supernatant instead of isolated messenger RNA (mRNA) for saliva transcriptomic detection (Lee et al., 2011). The validation was performed by means of reverse transcription quantitative polymerase chain reaction (RT-qPCR) in saliva taken from 30 gingivitis individuals in the clinical phase at $B, 3$ and 6 weeks. The $\mathrm{qPCR}$ associated with melting-curve analysis was conducted by the use of the LightCycler ${ }^{\circledR} 480$ Real-Time PCR System [Roche Life Science, Indianapolis, IN, USA] with a fixed thermal-cycling programme.

\subsubsection{2 | Statistical analysis}

Kruskal-Wallis and chi-squared tests were performed to compare markers between healthy and gingivitis groups in the discovery phase of transcriptomic analysis. In the validation phase, the analysis of variance (ANOVA) was done at a significance level of $p<0.05$. In addition, the logistic regression analysis in $\mathrm{R}$ was used to construct the final panel of biomarkers using the "best glm" function and the best Akaike Information Criterion. A paired $t$ test along with the $95 \%$ confidence interval $(\mathrm{Cl})$ was used to compare the Löe-Silness Gingival Index (GI) (Loe, 1967) and the Quigley and Hein Plaque Index (PI) (Turesky Modification) (Quigley \& Hein, 1962; Turesky, Gilmore, \& Glickman, 1970) scores over different time periods. Furthermore, generalized estimating equation
(GEE) models were added to show the overall trends in biomarker changes over time. Finally, inter-individual variability between two different clinical investigators was examined (the mean of each examiner's patients clinical scores at each time point). The examiners in the study followed the same patients through the investigation. The statistical analyses were performed using R V3.1.2 ( $\mathrm{R}$ Core Team, 2014) and SAS V9.4 (SAS Institute Inc., Cary, NC, USA), whereas the randomization was performed using the online statistical computing web programming "Sealed envelope" (https:// sealedenvelope.com).

\section{3 | RESULTS}

\section{1 | Clinical outcomes}

In the discovery phase, the study group consisted of 100 subjects, including 50 gingivitis subjects (22 males and 28 females) aged $26.4 \pm 6.77$ years, and 50 healthy individuals aged $27.1 \pm 5.67$ years (23 males and 27 females). The scores for the gingivitis group were $7.2 \pm 1.1 \%$ for Marginal Bleeding Index (MBI) (Loe, 1967; Muhlemann \& Son, 1971) and $2.5 \pm 0.7 \mathrm{~mm}$ for Probing Pocket Depth (PPD) (Ramfjord, 1959), while for the healthy group, the results were following: $2.4 \pm 1.6 \%$ for $\mathrm{MBI}$ and $2.1 \pm 0.8 \mathrm{~mm}$ for PPD. The participants were mainly of White origin (73\%), but also Asian (17\%), black or African American (10\%).

The validation phase included 30 subjects ( 13 males and 17 females), aged $28.2 \pm 7.77$ years. The participants were mainly of White origin (75\%), but also Asian (20\%) and black or African American (5\%). The average $\mathrm{Gl}$ scores were at Screening (Scr) $1.75 \pm 0.2$, at $\mathrm{B}-1.814 \pm 0.18$, at 3 weeks $-1.684 \pm 0.26$ and at 6 weeks $-1.46 \pm 0.55$, while the average PI scores were following: at Scr $2.293 \pm 0.44$, at B-2.307 \pm 0.34 , at 3 weeks $-2.054 \pm 0.33$ and at 6 weeks $-1.86 \pm 0.36$ (Supporting Information Table S1). Both GI and PI showed significantly decreased scores over time due to good oral hygiene regimens implemented by brushing with toothpastes (Figure 2). The $\mathrm{Gl}$ scores improved for $60 \%$ and PI for $93.3 \%$ of subjects over the period of 6 weeks.

Due to the long-term period of the study and large time difference between performing the discovery and the validation phases, there was a change in the current protocol for the enrolment of the patients into the study between the two stages. The MBI (Loe, 1967; Muhlemann \& Son, 1971) applied in the discovery phase, was replaced later, in the validation phase, with the more commonly used indices such as GI (Loe, 1967) and PI (Quigley \& Hein, 1962; Turesky et al., 1970) for the determination of the gingivitis status. The PPD (Ramfjord, 1959) was measured in both phases of the study. In the discovery phase, PPD was used to differentiate between healthy (non-periodontal disease) (PPD $<4 \mathrm{~mm}$ ) and gingivitis (PPD $<4 \mathrm{~mm}$ ) from periodontitis $(\geq 5 \mathrm{~mm}$ ) (apart from radiographic assessment). In the validation phase, only patients with diagnosed gingivitis were included. Study subjects could not have any PPD deeper than $5 \mathrm{~mm}$ (except for 3 rd molars) to be enrolled in the research project. 


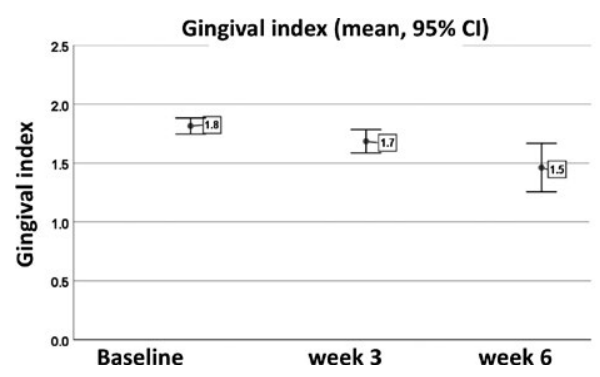

Baseline

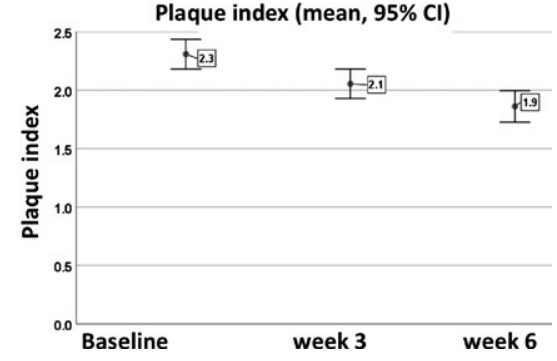

FIGURE 2 Comparison of the Gingival Index and the Plaque Index scores over time

\section{2 | Investigation of potential clinical evaluators bias}

There were no significant inter-examiner differences between the clinical evaluators, except for time point 6 weeks, which could potentially affect the GI and PI scores (Supporting Information Figure S1). This is despite the fact that all examiners were initially calibrated.

\section{3 | Discovery of salivary exRNA biomarker candidates for gingivitis by HTA Microarray profiling}

The 25 salivary exRNA biomarker candidates were identified from a discovery cohort of randomly selected 50 gingivitis and 50 age-/ gender-matched healthy controls (Table 1) (Figure 1). Of these, 10 differentially expressed exRNA biomarker candidates between gingivitis and healthy groups were advanced for validation by means of RT-qPCR using an independent cohort of 30 gingivitis subjects in the pilot clinical research study. Those exRNA biomarker candidates were selected based on their known association with inflammation and periodontal diseases, low $p$ value $(p<0.05)$ and high absolute fold change in their expression levels between gingivitis and healthy groups.

\section{4 | Validation of 10 salivary exRNA biomarker candidates for gingivitis by RT-qPCR}

Eight out of 10 validated exRNA targets were concordant with the HTA 2.0 microarray data (Table 2), including four decreased exRNAs [SPRR1A (m1), LGALS3 (m4), FAM25A (m7), CRCT1 (m10)] and four increased exRNAs [Inc-TET3-2:1 (m2), RP5965F6.2 (m3), GALNT10 (m5), SOX4 (m6)] over time (B-3 weeks, 3 weeks -6 weeks, B-6 weeks) (GEE $p$ values $<0.001$ ) (Supporting Information Figure S2, Supporting Information Table S1). Figure 3 presents a plot with fold changes and $95 \% \mathrm{Cls}$ for each marker at $\mathrm{B}$, 3 and 6 weeks. The increase in their alteration levels compared to the initial data might be a result of the inter-individual variability in exRNA patterns.

\section{5 | Logistic regression analysis}

The potential clinical discriminatory power of the developed salivary exRNA for gingivitis detection was evaluated. Logistic regression analysis of eight validated exRNAs revealed that four exRNA marker model [SPRR1A (m1), Inc-TET3-2:1 (m2), FAM25A (m7), CRCT1 (m10)] could potentially provide a discriminatory performance of 0.91 area under the curve with $71 \%$ sensitivity and $100 \%$ specificity (Figure 4).

\section{4 | DISCUSSION}

Saliva is a complex body fluid that is composed of unique molecular constituents (proteins, DNA, RNA, etc.) that can be discriminatory for oral and systemic disease screening, detection and monitoring (Kaczor-Urbanowicz et al., 2017). The development of non-invasive salivary test based on molecular biomarkers could have impactful clinical applications, providing accurate and real-time monitoring of periodontal disease activity for the general public at home or at the dental clinic. Although proteins are the essential effectors of cellular function, the eukaryotic complexity and phenotypic variation lies in a control of trans-acting RNAs-noncoding RNAs (ncRNAs) (Wong, 2015). NcRNAs regulate diverse biological functions, thus being very attractive for the development of new target therapies and discovery of new diagnostic biomarkers in human body fluids, including saliva (Ainsztein et al., 2015). The human salivary transcriptome was firstly described in 2004 (Li, Zhou, St John, \& Wong, 2004), followed by the intense investigations in the following years (Nussbaumer, Gharehbaghi-Schnell, \& Korschineck, 2006). The revealed complex exRNA composition of saliva encompasses mostly mRNAs, but also ncRNAs such as long noncoding RNAs (IncRNAs), microRNAs (miRNAs), small nucleolar RNAs (snoRNAs), piwi-interacting RNAs (piRNAs), etc. (Wong, 2015). Among all ncRNAs, small ncRNAs are the most exploited exRNAs in saliva, particularly miRNAs that play an important role in regulating various biological processes (Nelson, Kiriakidou, Sharma, Maniataki, \& Mourelatos, 2003), while the current knowledge of IncRNAs is still largely uninvestigated (Kung, Colognori, \& Lee, 2013).

The basis of biomarker research success in periodontology is to develop a diagnostic method that will have a potential to favourably affect clinical decision-making, patient outcomes and health care providers (Ghallab, 2017). Periodontal disease is time-consuming and expensive to treat, thus prevention and early detection constitutes significant health care benefit (Ghallab, 2017). Thus, it would be desirable to develop biomarkers for early detection and monitoring of periodontal disease and its treatment response (Giannobile et al., 2009). 


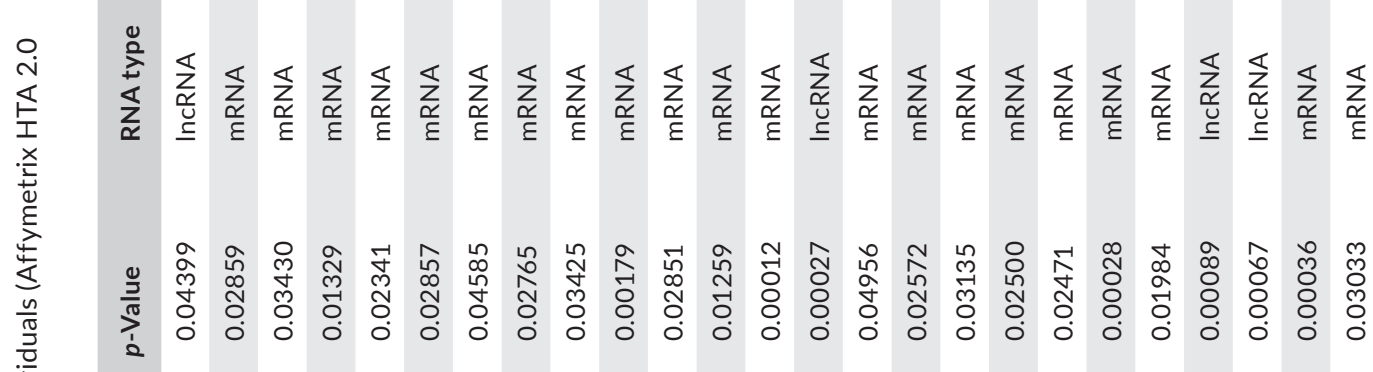

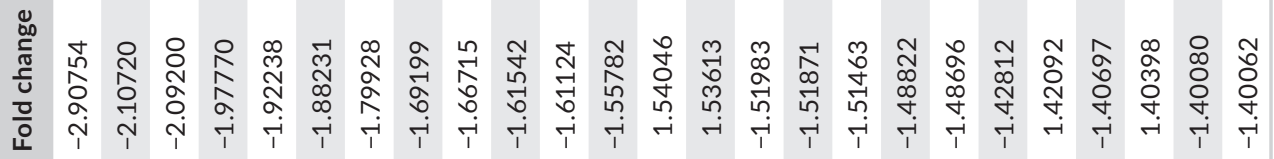

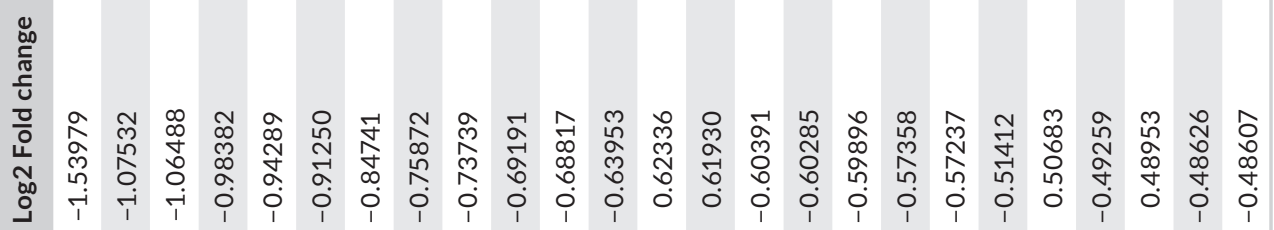

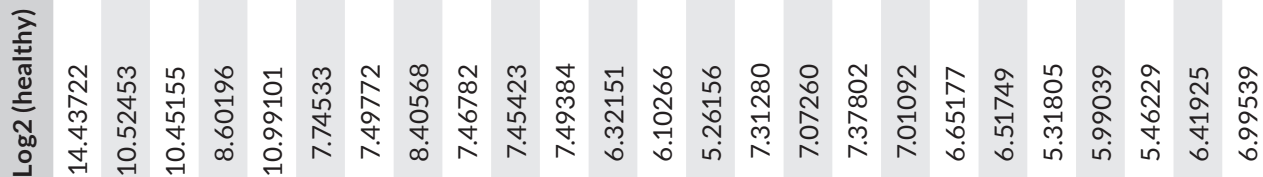

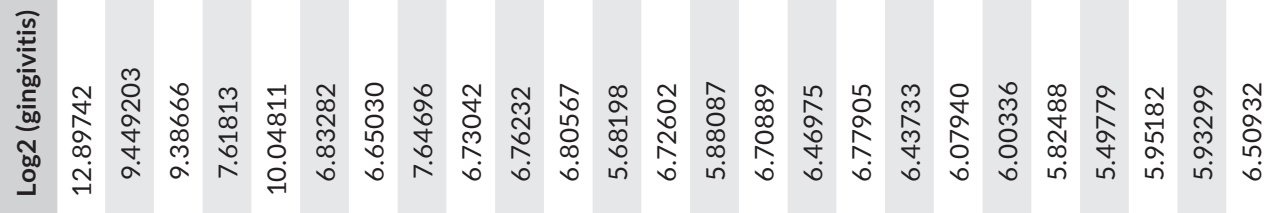

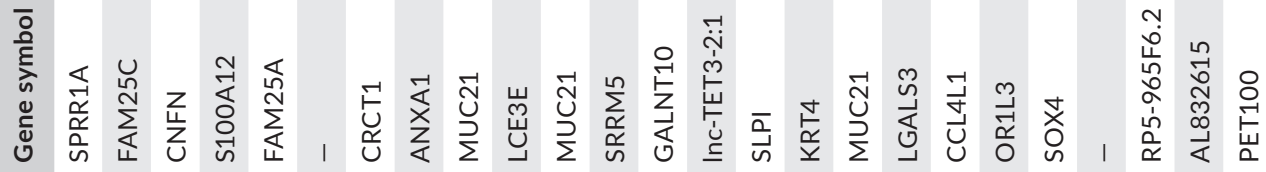

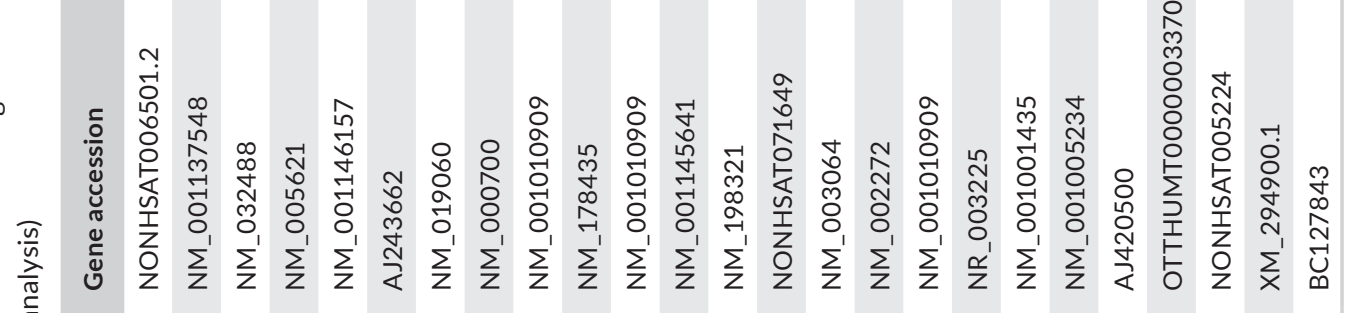
$\rightarrow>$

峞离

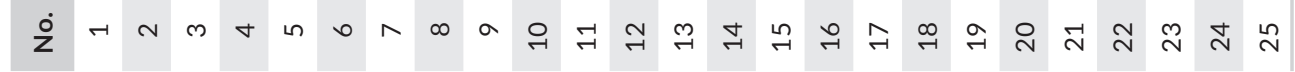


TABLE 2 Concordance of the 10 salivary exRNA biomarker changes with initial microarray data

\begin{tabular}{|c|c|c|c|c|c|c|}
\hline No. & Gene symbol & Gene accession & \multicolumn{4}{|c|}{ Association with disease } \\
\hline $\mathrm{m} 1$ & SPRR1A & NONHSAT006501.2 & - & 0.044 & - & $<0.001$ \\
\hline $\mathrm{m} 2$ & Inc-TET3-2:1 & NONHSAT071649 & + & $<0.001$ & + & $<0.001$ \\
\hline m5 & GALNT10 & NM_198321 & + & $<0.001$ & + & $<0.001$ \\
\hline $\mathrm{m} 6$ & sox4 & AJ420500 & + & 0.020 & + & $<0.001$ \\
\hline m7 & FAM25A & NM_001146157 & - & 0.023 & - & 0.003 \\
\hline $\mathrm{m} 8$ & AL832615 & XM_294900.1 & - & $<0.001$ & NS & 0.867 \\
\hline
\end{tabular}

Notes. Values reported as direction of effect \pm and $p$ value for statistical significance.

NS: nonsignificant.

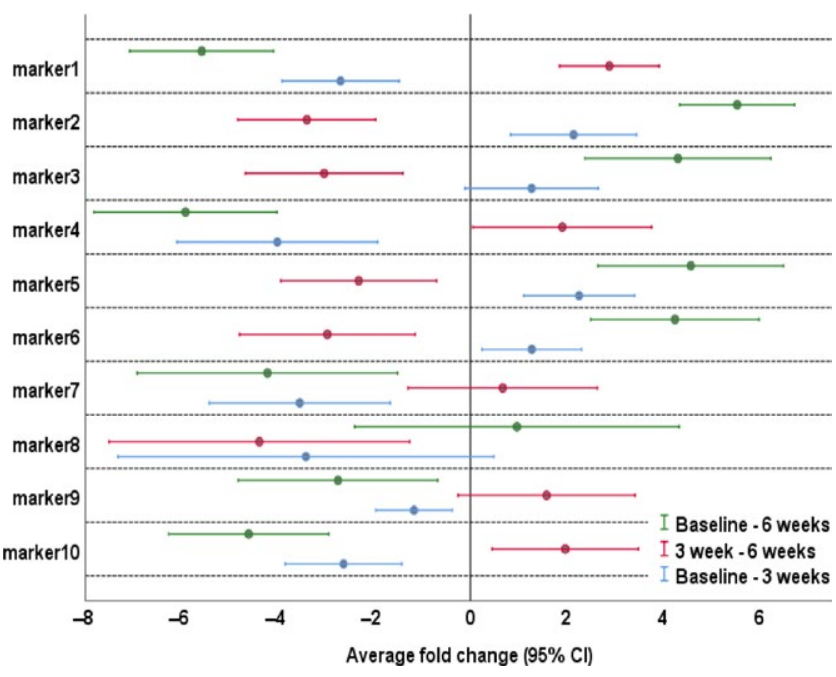

FIGURE 3 Validation of HTA 2.0 Affymetrix array profiling results by RT-qPCR. A plot presenting the fold changes and $95 \%$ confidence interval $(\mathrm{Cl})$ for each of 10 salivary exRNA biomarkers on the log base 2 scale for the following periods: baseline- 6 weeks, 3 week- 6 weeks and baseline -3 weeks

In the present study, the objective was to validate exRNA biomarkers for gingivitis, and to evaluate their potential to monitor disease regression. Eight salivary exRNAs showed significant positive correlation of their expression levels with improving clinical measures (GI, PI) due to the introduced treatment (brushing teeth), thus making them potentially useful in monitoring the current activity of disease and its treatment response. The identified exRNAs are mostly involved in inflammatory response, antimicrobial activity, innate immune response, apoptosis, neutrophil, eosinophil \& macrophage chemotaxis, and bone homeostasis, that is consistent with the current literature as periodontal diseases are mostly inflammatory in origin and involved in immune pathways (Ghallab, 2017) (Table 3).

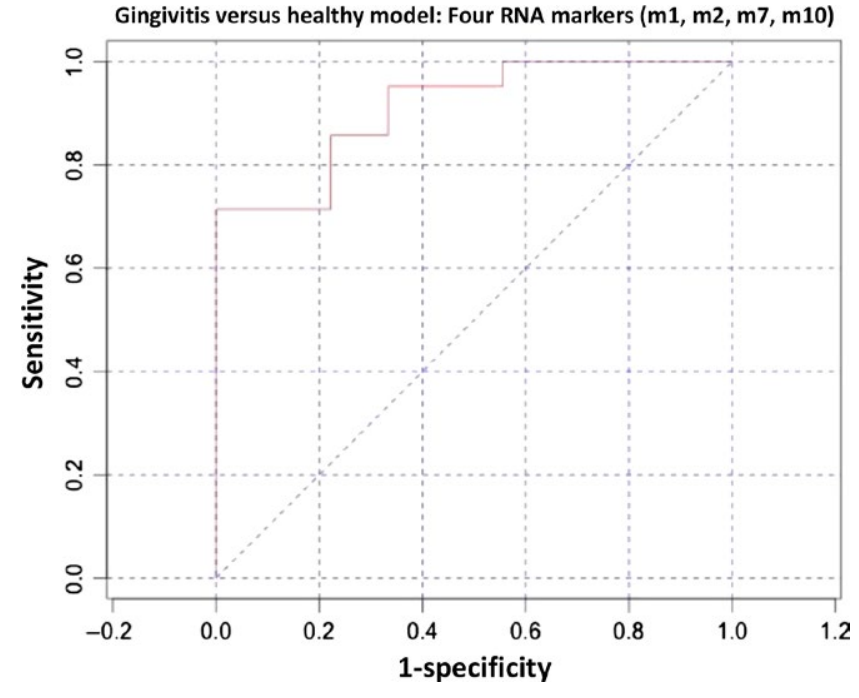

FIGURE 4 Performance of salivary exRNA biomarkers to classify gingivitis from healthy subjects with no periodontal disease (baseline-6 weeks) [4 exRNAs: SPRR1A (m1), Inc-TET3-2:1 (m2), FAM25A (m7), CRCT1 (m10)] (0.91 AUC, 71\% sensitivity, 100\% specificity)

Interestingly, only very few of them had been previously reported in different biofluids, but none of them have been identified in human saliva. Specifically, IncRNAs have aroused a lot of widespread interest in recent years as potentially associated in inducing particular diseases or developmental processes, but knowledge of the particular pathogenetic mechanisms by which they act is still limited. Therefore, it is not surprising that in the current literature, not much information can be found about the developed in this study-three IncRNA biomarkers of gingivitis (SPRR1A, Inc-TET3-2 and RP5965F6.2). It is known only that SPRR1A (m1) is a regenerationassociated gene (Starkey et al., 2009), whereas Inc-TET3-2:1 (m2) 
TABLE 3 Description of functions and biological roles of validated exRNAs for gingivitis

\begin{tabular}{|c|c|c|c|}
\hline No. & Gene symbol & Gene accession & Function/biological role \\
\hline $\mathrm{m} 1$ & SPRR1A & NONHSAT006501.2 & $\begin{array}{l}\text { Epidermatitis development, keratinocyte } \\
\text { differentiation and keratinization (Gibbs } \\
\text { et al., 1993; Stemmler et al., 2009) } \\
\text { Assessment of the regenerative potential of } \\
\text { injured neurons (Starkey et al., 2009) }\end{array}$ \\
\hline $\mathrm{m} 2$ & Inc-TET3-2:1 & NONHSAT071649 & $\begin{array}{l}\text { A member of the ten-eleven translocation } \\
\text { (TET) gene family, that plays a role in the } \\
\text { DNA methylation process (Langemeijer } \\
\text { et al., 2009) }\end{array}$ \\
\hline m3 & RP5-965F6.2 & NONHSAT005224 & $\begin{array}{l}\text { Has not been reported yet in the } \\
\text { publications }\end{array}$ \\
\hline m4 & LGALS3 & NR_003225 & $\begin{array}{l}\text { A member of the galectin family of } \\
\text { carbohydrate-binding proteins that play a } \\
\text { role in apoptosis, innate immunity, } \\
\text { neutrophil, eosinophil \& macrophage } \\
\text { chemotaxis and negative regulation of } \\
\text { endocytosis } \\
\text { Antimicrobial activity against bacteria and } \\
\text { fungi (http://www.genecards.org) (Raz } \\
\text { et al., 1991) } \\
\text { Association with increased risk for } \\
\text { rheumatoid arthritis (Atabaki, Hashemi, } \\
\text { Daneshvar, \& Alijani, 2017) }\end{array}$ \\
\hline m5 & $\begin{array}{l}\text { GALNT10 } \\
\text { AJ156166 }\end{array}$ & NM_198321 & $\begin{array}{l}\text { Homo sapiens putative tumour suppressor } \\
\text { mRNA (https://www.ncbi.nlm.nih.gov/ } \\
\text { nuccore) (Boultwood et al., 2000) }\end{array}$ \\
\hline m6 & sox4 & AJ420500 & $\begin{array}{l}\text { Apoptosis pathway leading to cell death as } \\
\text { well as to tumorigenesis } \\
\text { Mediation of downstream effects of } \\
\text { parathyroid hormone (PTH) and PTH- } \\
\text { related protein (PTHrP) in bone develop- } \\
\text { ment (http://www.genecards.org) } \\
\text { Obesity and type } 2 \text { diabetes (Ragvin et al., } \\
\text { 2010) } \\
\text { Airway inflammation (Kuwahara et al., } \\
\text { 2012) }\end{array}$ \\
\hline $\mathrm{m} 7$ & FAM25A & NM_001146157 & $\begin{array}{l}\text { Inflammation and infectious disease, } \\
\text { decrease in immune response (Deloukas } \\
\text { et al., 2004; Mauritz et al., 2010; Wang } \\
\text { et al., 2014) }\end{array}$ \\
\hline $\mathrm{m} 10$ & CRCT1 & NM_019060 & $\begin{array}{l}\text { Linkage to oesophageal cancer (Wu, Song, } \\
\text { Pang, \& Chen, 2016) and oropharyngeal } \\
\text { squamous cell carcinomas (Masterson } \\
\text { et al., 2015) } \\
\text { Early Alzheimer's disease-related stages } \\
\text { (Parra-Damas et al., 2014) } \\
\text { Promotion of tumour cell apoptosis and } \\
\text { upregulation of the expression of } \\
\text { apoptosis-related proteins (Wu et al., } \\
\text { 2016) }\end{array}$ \\
\hline
\end{tabular}

acts as an epigenetic mediator (Langemeijer, Aslanyan, \& Jansen, 2009) (Table 3).

In addition, our study also revealed five mRNA RNAs (LGALS3, GALNT10, SOX4, FAM25A and CRCT1) that are an emerging and flourishing field for non-invasive diagnostic applications. The identification of saliva-derived mRNA in normal and cancer patients (Hu et al., 2008; Li et al., 2004) and other forensic applications (Juusola \& Ballantyne, 2005) have opened up a new avenue for further clinical usage. According to the literature, LGALS3 (m4) can be informative on the inflammatory and bacterial nature of gingivitis due to their connectivity to the disease-related features such as its involvement in specific cellular (apoptosis, 
neutrophil, eosinophil and macrophage chemotaxis, etc.) and biological functions (antimicrobial activity, etc.) (Raz et al., 1991) (Table 3). As expected, in gingivitis due to gum inflammation, decreased levels of LGALS3 can be observed, that is concordant with our findings. In turn, alterations in SOX4 (m6) expression, induced by transforming growth factor beta, regulate T-helper type 2 (TH2) cell-driven airway inflammation (Kuwahara et al., 2012), whereas downregulation of FAM25A (m7) (observed also in our study) indicates increased risk for inflammation and infectious diseases (Deloukas et al., 2004; Mauritz et al., 2010; Wang, Kou, Wang, Cederbaum, \& Wang, 2014). Lastly, CRCT1 (m10) is reported to be associated with early stages of Alzheimer's disease, in which transcriptome changes affecting cell signalling, inflammation and neurotransmission pathways take place before the neuropathology in brains occur. In addition, the involvement of CRCT1 in protection against infection and inflammation is currently being explored (Parra-Damas et al., 2014) (Table 3). Thus, the potential role of the eight validated in this study exRNAs in pathogenetic mechanisms of periodontal diseases can be explained through their biological roles and potential functions (Table 3).

The major advantage of the study was the use of saliva, collected in a prospective way, as a potential non-invasive diagnostic source for detection and monitoring of gingivitis. Secondly, our study was focused on long RNAs as potential salivary biomarkers of periodontal diseases, including novel finding of IncRNAs, while the current literature reports mostly on short RNAs (microRNAs) with rather unsatisfactory performance (Kagiya, 2016; Ogata et al., 2014; Schmalz et al., 2016). Lastly, the use of DSTA method, that omits the step of RNA extraction, seems to be much more convenient and practical way of performing the experimental stage, as RNA isolation from saliva is not easy to perform and requires diligent protocol.

The main limitation of the study was the inter-individual differences in scoring of PI and GI, which suggest that a better system of clinical evaluation for gingivitis is largely needed, such as changes in expression levels of exRNA biomarkers, that can be used as companion diagnostic tool for periodontal and clinical examinations.

In addition, the same inclusion and exclusion criteria for clinical evaluation of the periodontal status (the same periodontal indices for both discovery and validation phases) are recommended to be used for the enrolment of the subjects into the project. Lastly, a comprehensive study of the variety of exRNAs and further increased sampling is needed to establish the sensitivity and specificity of the final panel of salivary exRNA biomarkers for gingivitis.

In fact, different groups have studied the exRNA composition of saliva for detection of periodontal diseases. Inflammatory mRNA markers for interferon gamma (IFN $\gamma$ ) and tumour necrosis factor alpha can be detected in whole saliva to monitor the status of periodontal disease in type II diabetes patients (Gomes et al., 2006). mRNA expression of human beta defensin-1 and -2 in the gingival tissue is associated with gingivitis, aggressive and chronic periodontitis (Vardar-Sengul et al., 2007). Also, the expression levels of Toll-like receptors (TLR)TLR2, -4, -7, -9, interferonalpha1 (IFN- $\alpha 1$ ) (Kajita et al., 2007) and MYD88 mRNAs were significantly lower in gingivitis than in severe periodontitis lesions (Ghaderi et al., 2014). In addition, four miRNAs (hsa-miR-451, hsa-miR-223, hsa-miR-486-5p and hsa-miR-3917) were significantly overexpressed, and seven (hsa-miR-1246, hsa-miR-1260, hsa-miR-141, hsa-miR-1260b, hsa-miR-203, hsa-miR-210 and hsamiR-205) were underexpressed by > twofold in a diseased compared to healthy gingiva (Stoecklin-Wasmer et al., 2012). In turn, Bochenek et al. (2013) report about downregulation of IncRNA ANRIL associated with atherosclerosis, periodontitis and several forms of cancer.

Several studies in the last decades have focused on finding a precise method for the diagnosis of periodontal disease in its early stages, such as gingivitis, as well as to monitor its treatment and progression into periodontitis. Interleukin 1 beta (IL-1 $\beta$ ) can be considered as an indicator of periodontal disease progression (Nazar Majeed, Philip, Alabsi, Pushparajan, \& Swaminathan, 2016), whereas matrix metalloproteinase-8 (MMP-8) may indicate response to therapy (Sexton et al., 2011). Additionally, macrophage inflammatory protein-1 $\alpha$ (MIP-1 $\alpha$ ) appeared to increase prediction of periodontitis progression (Fine et al., 2009) and to decrease its levels following periodontal therapy (Al-Sabbagh et al., 2012; Sexton et al., 2011).

Recent advances in transcriptomic high-throughput technologies are shedding a new light on salivary biomarker discovery, which can advance salivary diagnosis of periodontal diseases to a higher level. Salivary biomarkers for periodontal diseases need to have the intended clinical context to obtain approval from the Food and Drug Administration. The PRoBE design, applied in our study, greatly facilitates the future translation of the biomarkers into the everyday dental practice (Kim et al., 2013). Our results indicate that salivary exRNAs represent a promising source of biomarkers for gingivitis as well as monitoring disease regression. Although challenges emerge, using saliva seems to have a bright future in early detection of periodontal diseases and monitoring of periodontal treatment outcomes.

\section{CONFLICT OF INTEREST}

DTWW is co-founder of RNAmeTRIX Inc., a molecular diagnostic company. He holds equity in RNAmeTRIX, and serves as a company Director and Scientific Advisor. The University of California also holds equity in RNAmeTRIX. Intellectual property that David Wong invented and which was patented by the University of California has been licensed to RNAmeTRIX. Additionally, he is a consultant to GlaxoSmithKlein, Wrigley, EZLife Bio Inc. and ColgatePalmolive Company. WVG has previously consulted for Colgate, but not on this specific investigation. KEKU, HMT, JGM and DTWW have a pending patent related to this work [Attorney Docket No. 206030-0122-PI-US.606791]. None of the other authors have a conflict of interest to disclose related to this study. 


\section{ORCID}

Karolina E. Kaczor-Urbanowicz (iD http://orcid. org/0000-0001-9243-3652

William V. Giannobile (iD http://orcid.org/0000-0002-7102-9746

Carmen Martin Carerras-Presas (iD http://orcid. org/0000-0002-0937-0994

\section{REFERENCES}

Ainsztein, A. M., Brooks, P. J., Dugan, V. G., Ganguly, A., Guo, M., Howcroft, T. K., ... Venkatachalam, S. (2015). The NIH Extracellular RNA Communication Consortium. Journal of Extracellular Vesicles, 4, 27493. https://doi.org/10.3402/jev.v4.27493

Albandar, J. M., \& Kingman, A. (1999). Gingival recession, gingival bleeding, and dental calculus in adults 30 years of age and older in the United States, 1988-1994. Journal of Periodontology, 70, 30-43. https://doi.org/10.1902/jop.1999.70.1.30

Al-Sabbagh, M., Alladah, A., Lin, Y., Kryscio, R. J., Thomas, M. V., Ebersole, J. L., \& Miller, C. S. (2012). Bone remodeling-associated salivary biomarker MIP-1alpha distinguishes periodontal disease from health. Journal of Periodontal Research, 47, 389-395. https://doi. org/10.1111/j.1600-0765.2011.01445.x

Atabaki, M., Hashemi, M., Daneshvar, H., \& Alijani, E. (2017). Lectin, galactoside-binding, soluble, $3 \mathrm{rs} 4652 \mathrm{~A} / \mathrm{C}$ gene variation and the risk for rheumatoid arthritis. Biomedical Reports, 6, 251-255. https:// doi.org/10.3892/br.2017.838

Binder Gallimidi, A., Fischman, S., Revach, B., Bulvik, R., Maliutina, A., Rubinstein, A. M., ... Elkin, M. (2015). Periodontal pathogens Porphyromonas gingivalis and Fusobacterium nucleatum promote tumor progression in an oral-specific chemical carcinogenesis model. Oncotarget, 6, 22613-22623.

Bochenek, G., Hasler, R., El Mokhtari, N. E., Konig, I. R., Loos, B. G., Jepsen, S., ... Schaefer, A. S. (2013). The large non-coding RNA ANRIL, which is associated with atherosclerosis, periodontitis and several forms of cancer, regulates ADIPOR1, VAMP3 and C11ORF10. Human Molecular Genetics, 22, 4516-4527. https://doi.org/10.1093/ hmg/ddt299

Boultwood, J., Fidler, C., Strickson, A. J., Watkins, F., Kostrzewa, M., Jaju, R. J., ... Wainscoat, J. S. (2000). Transcription mapping of the 5q- syndrome critical region: Cloning of two novel genes and sequencing, expression, and mapping of a further six novel cDNAs. Genomics, 66, 26-34. https://doi.org/10.1006/geno.2000.6193

Chapple, I. L., Bouchard, P., Cagetti, M. G., Campus, G., Carra, M. C., Cocco, F., ... Schulte, A. G. (2017). Interaction of lifestyle, behaviour or systemic diseases with dental caries and periodontal diseases: Consensus report of group 2 of the joint EFP/ORCA workshop on the boundaries between caries and periodontal diseases. Journal of Clinical Periodontology, 44(Suppl 18), S39-S51. https://doi. org/10.1111/jcpe.12685

Deloukas, P., Earthrowl, M. E., Grafham, D. V., Rubenfield, M., French, L., Steward, C. A., ... Rogers, J. (2004). The DNA sequence and comparative analysis of human chromosome 10. Nature, 429, 375-381. https://doi.org/10.1038/nature02462

Ebersole, J. L., Nagarajan, R., Akers, D., \& Miller, C. S. (2015). Targeted salivary biomarkers for discrimination of periodontal health and disease(s). Frontiers in Cellular and Infection Microbiology, 5, 62.

Fine, D. H., Markowitz, K., Furgang, D., Fairlie, K., Ferrandiz, J., Nasri, C., ... Gunsolley, J. (2009). Macrophage inflammatory protein-1alpha: A salivary biomarker of bone loss in a longitudinal cohort study of children at risk for aggressive periodontal disease? Journal of Periodontology, 80, 106-113.
Ghaderi, H., Kiany, F., Razmkhah, M., Dadras, S., Chenari, N., Hosseini, A., ... Ghaderi, A. (2014). mRNA expression of pattern recognition receptors and their signaling mediators in healthy and diseased gingival tissues. Journal of Indian Society of Periodontology, 18, 150-154. https://doi.org/10.4103/0972-124X.131309

Ghallab, N. A. (2017). Diagnostic potential and future directions of biomarkers in gingival crevicular fluid and saliva of periodontal diseases: Review of the current evidence. Archives of Oral Biology, 87, 115-124.

Giannobile, W. V. (2012). Salivary diagnostics for periodontal diseases. Journal of the American Dental Association, 143, 6S-11S. https://doi. org/10.14219/jada.archive.2012.0341

Giannobile, W. V., Beikler, T., Kinney, J. S., Ramseier, C. A., Morelli, T., \& Wong, D. T. (2009). Saliva as a diagnostic tool for periodontal disease: Current state and future directions. Periodontology 2000, 50, 52-64. https://doi.org/10.1111/j.1600-0757.2008.00288.x

Gibbs, S., Fijneman, R., Wiegant, J., Van Kessel, A. G., Van De Putte, P., \& Backendorf, C. (1993). Molecular characterization and evolution of the SPRR family of keratinocyte differentiation markers encoding small proline-rich proteins. Genomics, 16, 630-637. https://doi. org/10.1006/geno.1993.1240

Gomes, M. A., Rodrigues, F. H., Afonso-Cardoso, S. R., Buso, A. M., Silva, A. G., Favoreto, Jr, S., \& Souza, M. A. (2006). Levels of immunoglobulin A1 and messenger RNA for interferon gamma and tumor necrosis factor alpha in total saliva from patients with diabetes mellitus type 2 with chronic periodontal disease. Journal of Periodontal Research, 41, 177-183. https://doi. org/10.1111/j.1600-0765.2005.00851.x

Grau, A. J., Becher, H., Ziegler, C. M., Lichy, C., Buggle, F., Kaiser, C., ... Dorfer, C. E. (2004). Periodontal disease as a risk factor for ischemic stroke. Stroke, 35, 496-501. https://doi.org/10.1161/01. STR.0000110789.20526.9D

Henskens, Y. M., Van Der Velden, U., Veerman, E. C., \& Nieuw Amerongen, A. V. (1993). Protein, albumin and cystatin concentrations in saliva of healthy subjects and of patients with gingivitis or periodontitis. Journal of Periodontal Research, 28, 43-48. https://doi. org/10.1111/j.1600-0765.1993.tb01049.x

Henson, B. S., \& Wong, D. T. (2010). Collection, storage, and processing of saliva samples for downstream molecular applications. Methods in Molecular Biology, 666, 21-30. https://doi. org/10.1007/978-1-60761-820-1

Hu, Z., Zimmermann, B. G., Zhou, H., Wang, J., Henson, B. S., Yu, W., ... Wong, D. T. (2008). Exon-level expression profiling: A comprehensive transcriptome analysis of oral fluids. Clinical Chemistry, 54, 824-832. https://doi.org/10.1373/clinchem.2007.096164

Juusola, J., \& Ballantyne, J. (2005). Multiplex mRNA profiling for the identification of body fluids. Forensic Science International, 152, 1-12. https://doi.org/10.1016/j.forsciint.2005.02.020

Kaczor-Urbanowicz, K. E., Martin Carreras-Presas, C., Aro, K., Tu, M., Garcia-Godoy, F., \& Wong, D. T. (2017). Saliva diagnostics-Current views and directions. Experimental Biology and Medicine (Maywood, N.J.), 242, 459-472. https://doi. org/10.1177/1535370216681550

Kagiya, T. (2016). MicroRNAs: Potential biomarkers and therapeutic targets for alveolar bone loss in periodontal disease. International Journal of Molecular Sciences, 17, 1317. https://doi.org/10.3390/ ijms17081317

Kajita, K., Honda, T., Amanuma, R., Domon, H., Okui, T., Ito, H., ... Yamazaki, K. (2007). Quantitative messenger RNA expression of Toll-like receptors and interferon-alpha1 in gingivitis and periodontitis. Oral Microbiology and Immunology, 22, 398-402. https://doi. org/10.1111/j.1399-302X.2007.00377.x

Kassebaum, N. J., Smith, A. G. C., Bernabe, E., Fleming, T. D., Reynolds, A. E., Vos, T., ... GBD 2015 Oral Health Collaborators. (2017). Global, regional, and national prevalence, incidence, and disability-adjusted life years for oral conditions for 195 countries, 1990-2015: A 
systematic analysis for the global burden of diseases, injuries, and risk factors. Journal of Dental Research, 96, 380-387. https://doi. org $/ 10.1177 / 0022034517693566$

Kim, J. J., Kim, C. J., \& Camargo, P. M. (2013). Salivary biomarkers in the diagnosis of periodontal diseases. Journal of the California Dental Association, 41, 119-124.

Kinney, J. S., Morelli, T., Braun, T., Ramseier, C. A., Herr, A. E., Sugai, J. V., ... Giannobile, W. V. (2011). Saliva/pathogen biomarker signatures and periodontal disease progression. Journal of Dental Research, 90, 752-758. https://doi.org/10.1177/0022034511399908

Kung, J. T., Colognori, D., \& Lee, J. T. (2013). Long noncoding RNAs: Past, present, and future. Genetics, 193, 651-669. https://doi.org/10.1534/ genetics.112.146704

Kuwahara, M., Yamashita, M., Shinoda, K., Tofukuji, S., Onodera, A., Shinnakasu, R., ... Nakayama, T. (2012). The transcription factor Sox4 is a downstream target of signaling by the cytokine TGF-beta and suppresses $\mathrm{T}(\mathrm{H}) 2$ differentiation. Nature Immunology, 13, 778-786. https://doi.org/10.1038/ni.2362

Langemeijer, S. M., Aslanyan, M. G., \& Jansen, J. H. (2009). TET proteins in malignant hematopoiesis. Cell Cycle, 8, 4044-4048. https://doi. org/10.4161/cc.8.24.10239

Lee, A., Ghaname, C. B., Braun, T. M., Sugai, J. V., Teles, R. P., Loesche, W. J., ... Kinney, J. S. (2012). Bacterial and salivary biomarkers predict the gingival inflammatory profile. Journal of Periodontology, 83, 79-89. https://doi.org/10.1902/jop.2011.110060

Lee, Y. H., Zhou, H., Reiss, J. K., Yan, X., Zhang, L., Chia, D., \& Wong, D. T. (2011). Direct saliva transcriptome analysis. Clinical Chemistry, 57, 1295-1302. https://doi.org/10.1373/clinchem.2010.159210

Li, Y., Zhou, X., St John, M. A., \& Wong, D. T. (2004). RNA profiling of cellfree saliva using microarray technology. Journal of Dental Research, 83, 199-203. https://doi.org/10.1177/154405910408300303

Loe, H. (1967). The Gingival Index, the Plaque Index and the Retention Index Systems. Journal of Periodontology, 38(Suppl), 610-616. https:// doi.org/10.1902/jop.1967.38.6_part2.610

Masterson, L., Sorgeloos, F., Winder, D., Lechner, M., Marker, A., Malhotra, S., ... Sterling, J. (2015). Deregulation of SYCP2 predicts early stage human papillomavirus-positive oropharyngeal carcinoma: A prospective whole transcriptome analysis. Cancer Science, 106, 1568-1575. https://doi.org/10.1111/cas.12809

Mauritz, J. M., Tiffert, T., Seear, R., Lautenschlager, F., Esposito, A., Lew, V. L., ... Kaminski, C. F. (2010). Detection of Plasmodium falciparuminfected red blood cells by optical stretching. Journal of Biomedial Optics, 15, 030517. https://doi.org/10.1117/1.3458919

Mitsuhashi, K., Nosho, K., Sukawa, Y., Matsunaga, Y., Ito, M., Kurihara, H., ... Shinomura, Y. (2015). Association of Fusobacterium species in pancreatic cancer tissues with molecular features and prognosis. Oncotarget, 6, 7209-7220.

Morelli, T., Stella, M., Barros, S. P., Marchesan, J. T., Moss, K. L., Kim, S. J., ... Offenbacher, S. (2014). Salivary biomarkers in a biofilm overgrowth model. Journal of Periodontology, 85, 1770-1778. https://doi. org/10.1902/jop.2014.140180

Muhlemann, H. R., \& Son, S. (1971). Gingival sulcus bleeding-A leading symptom in initial gingivitis. Helvetica Odontologica Acta, 15, 107-113.

Nazar Majeed, Z., Philip, K., Alabsi, A. M., Pushparajan, S., \& Swaminathan, D. (2016). Identification of gingival crevicular fluid sampling, analytical methods, and oral biomarkers for the diagnosis and monitoring of periodontal diseases: A systematic review. Disease Markers, 2016, 1804727.

Nelson, P., Kiriakidou, M., Sharma, A., Maniataki, E., \& Mourelatos, Z. (2003). The microRNA world: Small is mighty. Trends in Biochemical Sciences, 28, 534-540. https://doi.org/10.1016/j. tibs.2003.08.005

Nussbaumer, C., Gharehbaghi-Schnell, E., \& Korschineck, I. (2006). Messenger RNA profiling: A novel method for body fluid identification by real-time PCR. Forensic Science International, 157, 181-186. https://doi.org/10.1016/j.forsciint.2005.10.009

Ogata, Y., Matsui, S., Kato, A., Zhou, L., Nakayama, Y., \& Takai, H. (2014). MicroRNA expression in inflamed and noninflamed gingival tissues from Japanese patients. Journal of Oral Science, 56, 253-260. https:// doi.org/10.2334/josnusd.56.253

Parra-Damas, A., Valero, J., Chen, M., Espana, J., Martin, E., Ferrer, I., ... Saura, C. A. (2014). Crtc1 activates a transcriptional program deregulated at early Alzheimer's disease-related stages. Journal of Neuroscience, 34, 5776-5787. https://doi.org/10.1523/ JNEUROSCI.5288-13.2014

Pepe, M. S., Feng, Z., Janes, H., Bossuyt, P. M., \& Potter, J. D. (2008). Pivotal evaluation of the accuracy of a biomarker used for classification or prediction: Standards for study design. Journal of the National Cancer Institute, 100, 1432-1438. https://doi.org/10.1093/jnci/djn326

Quigley, G. A., \& Hein, J. W. (1962). Comparative cleansing efficiency of manual and power brushing. Journal of the American Dental Association, 65, 26-29. https://doi.org/10.14219/jada.archive.1962.0184

R Core Team. (2014). R: A language and environment for statistical computing. Vienna, Austria: R Foundation for Statistical Computing.

Ragvin, A., Moro, E., Fredman, D., Navratilova, P., Drivenes, O., Engstrom, P. G., ... Becker, T. S. (2010). Long-range gene regulation links genomic type 2 diabetes and obesity risk regions to HHEX, SOX4, and IRX3. Proceedings of the National Academy of Sciences of the United States of America, 107, 775-780. https://doi.org/10.1073/pnas.0911591107

Rajesh, K. S., Thomas, D., Hegde, S., \& Kumar, M. S. (2013). Poor periodontal health: A cancer risk? Journal of Indian Society of Periodontology, 17, 706-710.

Ramfjord, S. P. (1959). Indices for prevalence and incidence of periodontal disease. Journal of Periodontology, 30, 51-59. https://doi. org/10.1902/jop.1959.30.1.51

Raz, A., Carmi, P., Raz, T., Hogan, V., Mohamed, A., \& Wolman, S. R. (1991). Molecular cloning and chromosomal mapping of a human galactoside-binding protein. Cancer Research, 51, 2173-2178.

Schmalz, G., Li, S., Burkhardt, R., Rinke, S., Krause, F., Haak, R., \& Ziebolz, D. (2016). MicroRNAs as salivary markers for periodontal diseases: A new diagnostic approach? BioMed Research International, 2016, 1027525.

Sexton, W. M., Lin, Y., Kryscio, R. J., Dawson, 3rd, D. R., Ebersole, J. L., \& Miller, C. S. (2011). Salivary biomarkers of periodontal disease in response to treatment. Journal of Clinical Periodontology, 38, 434-441. https://doi.org/10.1111/j.1600-051X.2011.01706.x

Shaila, M., Pai, G. P., \& Shetty, P. (2013). Salivary protein concentration, flow rate, buffer capacity and $\mathrm{pH}$ estimation: A comparative study among young and elderly subjects, both normal and with gingivitis and periodontitis. Journal of Indian Society of Periodontology, 17, 4246. https://doi.org/10.4103/0972-124X.107473

Starkey, M. L., Davies, M., Yip, P. K., Carter, L. M., Wong, D. J., Mcmahon, S. B., \& Bradbury, E. J. (2009). Expression of the regenerationassociated protein SPRR1A in primary sensory neurons and spinal cord of the adult mouse following peripheral and central injury. The Journal of Comparative Neurology, 513, 51-68. https://doi. org/10.1002/cne.21944

Stemmler, S., Nothnagel, M., Parwez, Q., Petrasch-Parwez, E., Epplen, J. T., \& Hoffjan, S. (2009). Variation in genes of the epidermal differentiation complex in German atopic dermatitis patients. International Journal of Immunogenetics, 36, 217-222. https://doi. org/10.1111/j.1744-313X.2009.00858.x

Stoecklin-Wasmer, C., Guarnieri, P., Celenti, R., Demmer, R. T., Kebschull, M., \& Papapanou, P. N. (2012). MicroRNAs and their target genes in gingival tissues. Journal of Dental Research, 91, 934-940. https://doi. org/10.1177/0022034512456551

Tomar, S. L., \& Asma, S. (2000). Smoking-attributable periodontitis in the United States: Findings from NHANES III. National Health and Nutrition Examination Survey. Journal of Periodontology, 71, 743-751. https://doi.org/10.1902/jop.2000.71.5.743 
Turesky, S., Gilmore, N. D., \& Glickman, I. (1970). Reduced plaque formation by the chloromethyl analogue of victamine C. Journal of Periodontology, 41, 41-43. https://doi.org/10.1902/jop.1970.41.41.41

Vardar-Sengul, S., Demirci, T., Sen, B. H., Erkizan, V., Kurulgan, E., \& Baylas, H. (2007). Human beta defensin-1 and -2 expression in the gingiva of patients with specific periodontal diseases. Journal of Periodontal Research, 42, 429-437. https://doi. org/10.1111/j.1600-0765.2006.00964.x

Wang, Y., Kou, Y., Wang, X., Cederbaum, A., \& Wang, R. (2014). Multifactorial comparative proteomic study of cytochrome P450 2E1 function in chronic alcohol administration. PLoS ONE, 9, e92504. https://doi.org/10.1371/journal.pone.0092504

Wong, D. T. (2015). Salivary extracellular noncoding RNA: Emerging biomarkers for molecular diagnostics. Clinical Therapeutics, 37, 540-551. https://doi.org/10.1016/j.clinthera.2015.02.017

Wu, N., Song, Y., Pang, L., \& Chen, Z. (2016). CRCT1 regulated by microRNA-520 g inhibits proliferation and induces apoptosis in esophageal squamous cell cancer. Tumour Biology, 37, 8271-8279. https://doi. org/10.1007/s13277-015-4730-2

\section{SUPPORTING INFORMATION}

Additional supporting information may be found online in the Supporting Information section at the end of the article.

How to cite this article: Kaczor-Urbanowicz KE, Trivedi HM, Lima PO, et al. Salivary exRNA biomarkers to detect gingivitis and monitor disease regression. J Clin Periodontol. 2018;45:806817. https://doi.org/10.1111/jcpe.12930 